\title{
Foreword to the special issue on nonlinear waves over variable bathymetry
}

\author{
G. A. Athanassoulis ${ }^{1} \cdot$ M. Benoit ${ }^{2} \cdot$ D. Clamond $^{3} \cdot$ S. T. Grilli ${ }^{4}$
}

Published online: 23 January 2020

○) Springer Nature Switzerland AG 2020

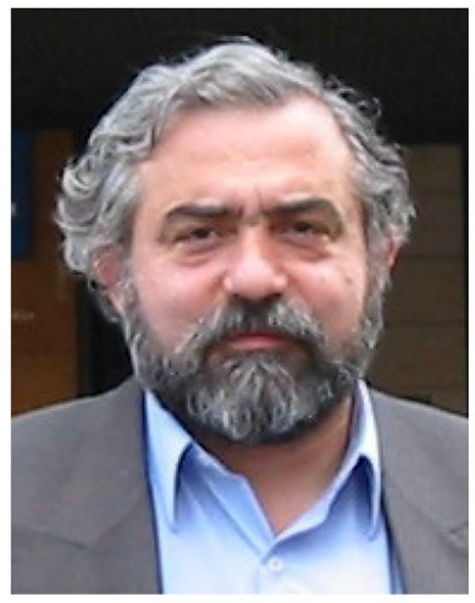

G.A. Athanassoulis

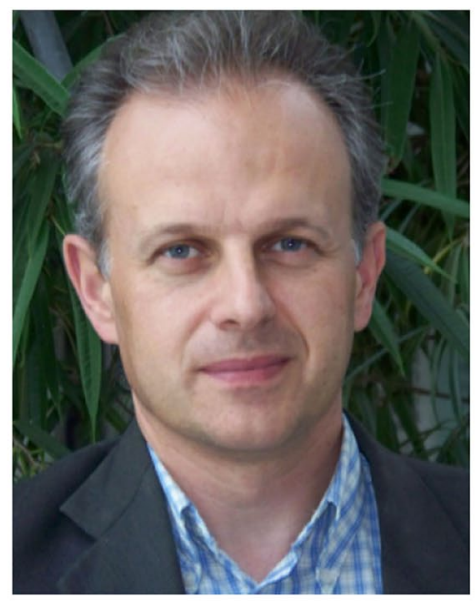

M. Benoit

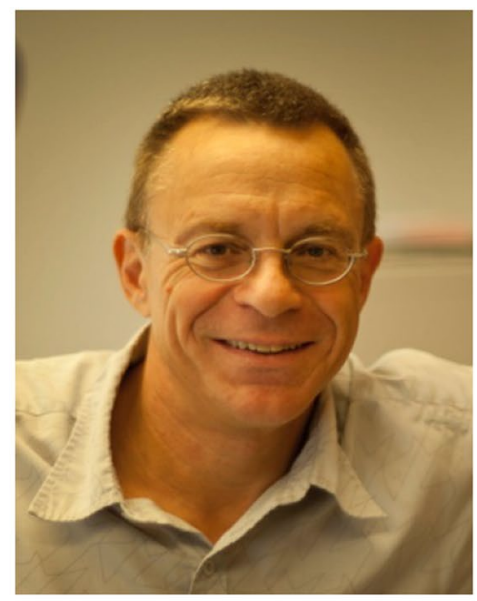

S.T. Grilli
In the last few decades, various methods have been developed for the accurate numerical treatment of fully nonlinear free-surface problems in water of varying bathymetry. Most of these methods are based on potential theory, which excludes the effects of viscosity and irrotationality. Important examples of direct numerical approaches are the methods of finite differences (e.g., Bingham and Zhang 2007; Ducrozet et al. 2010), and finite elements (e.g., Ma and Yan 2006; Gagarina et al. 2014), that require the construction of computational grids covering the entire fluid domain. An alternative line of work is based on the Hamiltonian

G. A. Athanassoulis

mathan@central.ntua.gr

1 Technical University of Athens, Athens, Greece

2 Institut de Recherche sur les Phénomènes Hors Équilibre and École Centrale Marseille, Marseille, France

3 University of Nice - Sophia Antipolis, Nice, France

4 University of Rhode Island, Kingston, USA (re)formulation of the problem. In this direction, the fully nonlinear problem is recast as a system of two canonical dynamical equations, coupled with a kinematic problem describing the vertical structure of the substrate flow. The latter problem can be formulated as an appropriate Dirichletto-Neumann (DtN) operator. DtN operator can be approximated with a Fréchet-Taylor series expansion (Craig and Sulem 1993; Craig et al. 2005), or can be calculated using boundary elements (e.g., Grilli et al. 2001; Fructus et al. 2005; Fochesato and Dias 2006; Guyenne and Grilli 2006). Alternatively, it can be treated using an appropriate expansion of the vertical structure of the wave flow, with coefficients obtained by solving an auxiliary, kinematical problem. Variants of the latter approach include the Hamiltonian coupled-mode theory (HCMT) (Athanassoulis and Papoutsellis 2017; Papoutsellis et al. 2018), higher order spectral methods (e.g. Liu and Yue 1998; Gouin et al. 2016), the spectral approach based on Chebyshev polynomials (Yates and Benoit 2015; Benoit et al. 2017; Raoult et al. 2019), and 
K-level irrotational Green-Naghdi (IGN-K) methods (e.g. Zhao et al. 2015) ${ }^{1}$

In addition, various model equations based on vertical averaging of the flow or/and perturbation analyses such as, e.g., Boussinesq-type equations (e.g. Madsen and Schäffer 1998; Madsen et al. 2002; Kirby 2016), low-level IGN equations, and nonlinear variants of multimodal coupledmode equations, are available and widely used in engineering applications involving varying bathymetry coastal environments. Also, simplified model equations, e.g., Benney-Roskes (BR) and nonlinear Schrödinger (NLS) equations (e.g., Djordjevic and Redekopp 1978; Zeng and Trulsen 2012), are used for studying stability aspects of wave trains.

Challenging problems in water-wave dynamics go beyond potential flow theory, calling for the inclusion of rotational and viscous effects. Attempts to address these features have already appeared and are expected to become predominant in the near future.

The present special issue contains eight papers in total: three papers applying advanced, potential theory-based methods to wave problems involving complicated, uneven seabeds (Athanassoulis et al. 2019; Touboul and Belibassakis 2019; Zhao et al. 2019) ${ }^{2}$; a paper exploiting a fully nonlinear potential flow solver to model wave breaking by means of boundary dissipation (Simon et al. 2019); two papers investigating how simplified model equations can be best adapted to varying bathymetry conditions (Lalli et al. 2019; Yu 2019); a paper examining the effect of wind forcing on modulational instability (Grimshaw 2019); and another one using the Navier-Stokes equations, thus being capable of producing reliable results for complicated nonlinear wave-seabed interactions, including rotational waveinduced currents (Gallerano et al. 2019). More specifically:

Athanassoulis et al. (2019) study the nonlinear interaction of a solitary wave with a bathymetry having one or two tandem trenches, using the Hamiltonian coupled-mode theory (HCMT). In this approach, the vertical structure of the wave potential is exactly represented by a local vertical basis, and the fully nonlinear wave problem is reformulated as two Hamiltonian evolution equations with respect to the surface elevation and the surface potential, coupled with a system of instantaneous yet time-independent horizontal equations, modelling the substrate kinematics. The solitary wave, after its transformation by the trenches, impinges on a vertical wall. The run-up and the force exerted on the wall are calculated and compared with the corresponding ones in the case of a flat bathymetry. The significant reduction of

\footnotetext{
1 The literature on these topics is vast. The references given herein are just indicative.

${ }^{2}$ See the list of papers contained in the present special issue, at the end of this foreword.
}

both the run-up and the force, when the trenches are present, indicates the usefulness of this bathymetry as a submerged breakwater.

Gallerano et al. (2019) investigate the effect of nonlinear interaction of surface waves with complicated bathymetries, and especially a system of submerged breakwaters. They use an integral, contravariant form of the Navier-Stokes equations, in time-dependent, curvilinear coordinates, allowing for the discretization of the irregular physical domain by curvilinear cells, adapted to the free-surface evolution. The use of a time-dependent coordinate transformation leads to finite-volume equations of motion in the transformed space, where the computational cells remain regular and fixed. The integration is performed using a shock-capturing scheme permitting to treat wave breaking as well. The generality of their model permits the authors to produce reliable results for wave motion, mean water level, and wave-induced circulation patterns. The latter are indispensable for the assessment of various coastal structures, regarding their erosive or accretive action.

Grimshaw (2019) investigates the effect of wind forcing on the instability of water waves in two horizontal space dimensions, over a seabed of finite depth. The stability analysis is performed on a forced version of the Benney-Roskes (BR) equations, obtained by introducing a wind forcing in terms of a linear growth rate term. Also, considering the one-dimensional reduction of BR equations to NLS equation, he examines the effect of wind forcing on a Peregrine breather. The main conclusions of the analysis presented in this paper are: wind forcing enhances modulation instability, rendering it super-exponential in two dimensions, and the domain of instability in the modulation wavenumber space is enlarged. Also, it is found that, for the case of a Peregrine breather, the wind forcing leads to an exponential amplitude growth at a rate twice that of the linear instability analysis.

Lalli et al. (2019) develop a generalization of Green's law, for long waves, by means of a semi-analytical approach that involves the numerical calculation of a parameter, which depends on the morphology of the seabed. In this way, they are able to describe the amplitude modulation of long waves approaching coasts of complex bathymetry, accounting not only for the wave shoaling, but also for the refraction and diffraction phenomena, as well. They present comparisons of the proposed method with numerical results, obtained by means of a solver based on the shallow-water equations. Their conclusion is that the proposed generalized Green's law has an overall good performance when complex bathymetries are considered, except for small areas near seabed locations where the diffraction is intense.

Simon et al. (2019) are concerned with the modelling of wave-breaking onset and dissipation, in the context of a fully nonlinear and dispersive potential flow model. Nonlinear wave evolution is described by the Hamiltonian formulation 
treated with a spectral representation of the vertical structure of wave flow, using Chebyshev polynomials. Dissipation is introduced in the form of a surface pressure term, activated by appropriate wave-breaking criteria. Three criteria concerning the onset of wave breaking and three dissipation methods are implemented and tested against laboratory experiments: regular and irregular waves breaking over a submerged bar, irregular waves breaking on a plane beach, and irregular waves breaking over a submerged slope. Different combinations of criteria and dissipative terms provide similar results. In general, wave transformation and post-breaking evolution are well reproduced, as it is shown by comparisons of wave statistics and wave spectra with experimental results.

Touboul and Belibassakis (2019) investigate the problem of water waves propagating over variable bathymetry in the presence of vertically sheared currents. They use a coupled-mode model to decompose the velocity field to a mean current and a wave flow, the latter being expressed by a series of local vertical modes analytically treating the continuity equation and the bottom boundary condition on the sloping parts of the seabed. Under the assumption of small amplitude waves, the system is linearized and its dispersion characteristics are studied for general vertical distribution of the sheared current. The structure of the model facilitates future extensions to three-dimensional configurations and the inclusion of nonlinear effects.

$\mathrm{Yu}$ (2019), using the conformal mapping technique, rederives the terrain-following Boussinesq-type equations for an arbitrarily periodic bed, and points out important issues regarding the existing approach. Specifically, she clarifies the definition of the velocity vector in the mapped plane, and, based on results of the exact Floquet theory, demonstrates that the linear dispersion relation is modified due to the presence of periodic seabed. Also, she examines the waveforms of linear time harmonic waves over a highly variable periodic seabed, noting features that resemble nonlinear wave motion. The theoretical results of this paper clarify various aspects of the nonlinear, Boussinesq-type equations over arbitrarily periodic beds, paving the way to a more efficient exploitation of the latter.

Zhao et al. (2019) investigate the performance of Irrotational Green-Naghdi II (ING-2) equations in solving threedimensional, strongly nonlinear and dispersive, free-surface wave problems over uneven bathymetry. The wave flow is represented by a stream function assumed to have a polynomial vertical structure. The equations of motion, obtained via Hamilton's principle, include two canonical dynamical equations with respect to the surface elevation and the surface potential, and a set of horizontal equations describing the instantaneous substrate kinematics, similarly as in the HCMT. The authors, after testing successfully the method in some simple cases, they apply it to the demanding problem of wave diffraction due to a three-dimensional circular shoal. The obtained numerical results are in very good agreement with numerical results from highly accurate Boussinesq equations and experimental measurements. They conclude that the IGN-2 equations may be more suitable, for many coastal engineering problems, than a number of other perturbation-based methods, because of the simplicity of the theory.

We hope that this special issue, exploiting some of the best nonlinear methods for water waves over varying bathymetry, and providing new ideas and new insights into important specific directions, will be valuable to researchers and practitioners dealing with problems in coastal environments.

Guest Editors of the Special Issue

G. A. Athanassoulis

M. Benoit

D. Clamond

S. T. Grilli

\section{References}

Athanassoulis GA, Papoutsellis CE (2017) Exact semi-separation of variables in waveguides with non-planar boundaries. Proc R Soc A Math Phys Eng Sci 473(2201):20170017

Athanassoulis GA, Mavroeidis CP, Koutsogiannakis PE, Papoutsellis CE (2019) A numerical study of the run-up and the force exerted on a vertical wall by a solitary wave propagating over two tandem trenches. J Ocean Eng Mar Energy (this issue)

Benoit M, Raoult C, Yates ML (2017) Analysis of the linear version of a highly dispersive potential water wave model using a spectral approach in the vertical. Wave Motion 74:159-181

Bingham HB, Zhang H (2007) On the accuracy of finite-difference solutions for nonlinear water waves. J Eng Math 58(1):211-228

Craig W, Sulem C (1993) Numerical simulation of gravity waves. J Comput Phys 108(1):73-83

Craig W, Guyenne P, Nicholls DP, Sulem C (2005) Hamiltonian longwave expansions for water waves over a rough bottom. Proc R Soc A Math Phys Eng Sci 461(2055):839-873

Djordjevic VD, Redekopp LG (1978) On the development of packets of surface gravity waves moving over an uneven bottom. J Appl Math Phys 29:950-962

Ducrozet G, Bingham HB, Engsig-Karup AP, Ferrant P (2010) Highorder finite difference solution for 3D nonlinear wave-structure interaction. J Hydrodyn 22(1):225-230

Fochesato C, Dias F (2006) A fast method for nonlinear three-dimensional free-surface waves. Proc R Soc A Math Phys Eng Sci 462(2073):2715-2735

Fructus D, Clamond D, Grue J, Kristiansen Ø (2005) An efficient model for three-dimensional surface wave simulations: part i: free space problems. J Comput Phys 205(2):665-685

Gagarina E, Ambati VR, van der Vegt JJW, Bokhove O (2014) Variational space-time (dis)continuous galerkin method for nonlinear free surface water waves. J Comput Phys 275:459-483

Gallerano F, Cannata G, Palleschi F (2019) Nonlinear waves and nearshore currents over variable bathymetry in curved shaped coastal areas. J Ocean Eng Mar Energy (this issue) 
Gouin M, Ducrozet G, Ferrant P (2016) Development and validation of a non-linear spectral model for water waves over variable depth. Eur J Mech B Fluids 57:115-128

Grilli S, Guyenne P, Dias F (2001) A fully non-linear model for threedimensional overturning waves over an arbitrary bottom. Int $\mathbf{J}$ Numer Methods Fluids 35(7):829-867

Grimshaw R (2019) Two-dimensional modulation instability of wind waves. J Ocean Eng Mar Energy (this issue)

Guyenne P, Grilli ST (2006) Numerical study of three-dimensional overturning waves in shallow water. J Fluid Mech 547:361-388

Kirby JT (2016) Boussinesq models and their application to coastal processes across a wide range of scales. J Waterw Port Coast Ocean Eng 142(6):03116005

Lalli F, Postacchini M, Brocchini M (2019) Long waves approaching the coast: Green's law generalization. J Ocean Eng Mar Energy (this issue)

Liu Y, Yue DKP (1998) On generalized bragg scattering of surface waves by bottom ripples. J Fluid Mech 356:297-326

Ma QW, Yan S (2006) Quasi ALE finite element method for nonlinear water waves. J Comput Phys 212(1):52-72

Madsen PA, Schäffer HA (1998) Higher order Boussinesq-type equations for surfacegravity waves: derivation and analysis. Philos Trans R Soc A 356:3123-3181

Madsen PA, Bingham HB, Liu H (2002) A new Boussinesq method for fully nonlinear waves from shallow to deep water. J Fluid Mech 462:1-30

Papoutsellis CE, Charalampopoulos AG, Athanassoulis GA (2018) Implementation of a fully nonlinear hamiltonian coupled-mode theory, and application to solitary wave problems over bathymetry. Eur J Mech B Fluids 72:199-224
Raoult C, Benoit M, Yates ML (2019) Development and validation of a 3D RBF-spectral model for coastal wave simulation. J Comput Phys 378:278-302

Simon B, Papoutsellis CE, Benoit M, Yates ML (2019) Comparing methods of modeling depth-induced breaking of irregular waves with a fully nonlinear potential flow approach. J Ocean Eng Mar Energy (this issue)

Touboul J, Belibassakis K (2019) A novel method for water waves propagating in the presence of vortical mean flows over variable bathymetry. J Ocean Eng Mar Energy (this issue)

Yates ML, Benoit M (2015) Accuracy and efficiency of two numerical methods of solving the potential flow problem for highly nonlinear and dispersive water waves. Int J Numer Methods Fluids 77(10):616-640

Yu J (2019) Revisiting terrain-following Boussinesq equations on a highly variable periodic bed. J Ocean Eng Mar Energy (this issue)

Zeng H, Trulsen K (2012) Evolution of skewness and kurtosis of weakly nonlinear unidirectional waves over a sloping bottom. Nat Hazards Earth Syst Sci 12:631-638. http://www.nat-hazards-earth -syst-sci.net/12/631/2012/

Zhao BB, Duan WY, Ertekin RC, Hayatdavoodi M (2015) High-level Green-Naghdi wave models for nonlinear wave transformation in three dimensions. J Ocean Eng Mar Energy 1(2):121-132

Zhao BB, Zhang T, Duan W, Ertekin RC, Hayatdavoodi M (2019) Application of three-dimensional IGN-2 equations to wave diffraction problems. J Ocean Eng Mar Energy (this issue)

Publisher's Note Springer Nature remains neutral with regard to jurisdictional claims in published maps and institutional affiliations. 